\title{
Bilirubin Interactions with Phospholipid Components of Lung Surfactant
}

\author{
R. H. NOTTER, ${ }^{(23)}$ D. L. SHAPIRO, R. TAUBOLD AND J. CHEN \\ Division of Neonatology, Department of Pediatrics, University of Rochester School of Medicine, Rochester, New York, \\ USA
}

\begin{abstract}
Summary
This work examines the dynamic surface pressure-area behavior of films of unconjugated bilirubin spread from chloroform solution at $22^{\circ} \mathrm{C}$ on $0.15 \mathrm{M} \mathrm{NaCl}$ and buffered phosphate subphases. Film behavior is examined at $\mathrm{pH} 5.5,7.4$ and 8.0 . The interactions of bilirubin in mixed films with dipalmitoyl phosphatidylcholine and with 9:1 dipalmitoyl phosphatidylcholine:dioleolyl phosphatidylcholine are examined at similar temperature and $\mathrm{pH}$ values. It is found that unconjugated bilirubin modifies the dynamic surface pressure-area behavior of phospholipid films in both the high and low surface pressure regimes, with bilirubin exerting its greatest effect at low pH where its subphase solubility is low. Because many premature infants suffering from the Respiratory Distress Syndrome (RDS) have accompanying hyperbilirubinemia, with possible bilirubin transport to the alveolar space, the interactions of bilirubin with phospholipid films are discussed in terms of potential effects on pulmonary surfactant in vivo.
\end{abstract}

\section{Speculation}

In the presence of hyperbilirubinemia in the neonate, bilirubin can enter the alveolar space and has the potential to affect the properties of pulmonary surfactant. Surface balance studies show no clear detrimental effect of bilirubin on phospholipid films in terms of inhibiting physiologically relevant surface properties such as dynamic surface tension lowering or respreading capability. Alveolar bilirubin by itself does not appear to contribute to pulmonary pathophysiology in terms of surface tension effects.

Broderson (4) has recently reviewed the current knowledge and extensive ongoing research concerning bilirubin transport and toxicity in the neonate. Most interest in hyperbilirubinemia in the newborn period has centered on the toxic effects of the acid form of bilirubin to the central nervous system. By contrast, little work has been directed at investigating other organ systems where high serum concentrations of bilirubin might play a pathologic role. During hyperbilirubinemia in the newborn period, bilirubin distributes to many tissues (2). It apparently can enter the alveolar space, probably in fluid leaked from capillaries, where its presence at autopsy has been termed "Yellow Hyaline Membrane Disease". However, there is no information on whether this pathologic entity reflects disordered pulmonary physiology in the living newborn infant.

It is reasonable to ask if bilirubin interacts with lung surfactant or with lung surfactant components in terms of physiologically detrimental effects on surface activity. If true, this might have therapeutic implications for clinical management, prompting a more aggressive approach to hyperbilirubinemia in the presence of RDS. The present work studies interactions between bilirubin and lung surfactant components in several well-defined surface films as an initial attempt to see if bilirubin might have significant effects on surface tension lowering behavior. Specifically, we investigate the surface interactions of bilirubin with dipalmitoyl phosphatidylcholine (DPPC) and dioleoyl phosphatidylcholine (DOPC). DPPC is the primary phospholipid component of lung surfactant and is widely held to be responsible for the extremely effective surface tension lowering effects of the natural system (e.g., 7, 13). DOPC is an unsaturated phospholipid, also present in lung extracts, which confers better adsorption and dynamic respreading properties in mixed films with DPPC (9).

Little work has been done on the surface tension lowering properties of porphyrin derivatives. Alexander (1) studied the surface behavior of several porphyrins in 1937, and several years later Stenhagen and Rideal (16) examined the interactions of bilirubin and other porphyrins with monolayers of cholesterol and protein in terms of surface tension and surface potential effects. Both of these early studies $(1,16)$ were done with a Langmuir Trough, and did not examine film behavior under continuous dynamic compression. For applications to pulmonary surfactant, the effects of bilirubin on DPPC and DPPC:DOPC films in terms of dynamic surface tension lowering behavior are of primary interest in the physiologic sense. No previous work of this type is available. Thus, the present study concentrates on delineating the dynamic surface pressure-area ( $\pi-A)(19)$ behavior of unconjugated bilirubin in pure films, and in mixed films with DPPC and 9:1 DPPC:DOPC (molar ratio). Experiments are also done as a function of subphase $\mathrm{pH}$ because of its significant effect on bilirubin properties.

\section{MATERIALS AND METHODS}

Bilirubin (B Grade, Lot 902545, Molecular weight 585) was obtained from Calbiochem (LaJolla, CA). Dipalmitoyl-3-sn-phosphatidylcholine (DPPC) and dioleoyl-3-sn-phosphatidylcholine (DOPC) were purchased from the Sigma Chemical Co. (St. Louis, MO), and from Supelco (Bellefonte, PA), respectively. The phospholipids were found pure by thin layer chromatography, with $>99 \% \mathrm{C} 16: 0$ or $\mathrm{C} 18: 1$ chains by gas chromatography. The extinction coefficient of the bilirubin at $454 \mathrm{~nm}$ was measured as $\epsilon=$ $6.05 \times 10^{4}$, compared to a value of $6.04 \times 10^{4}$ furnished by Calbiochem. All three compounds, DPPC, DOPC, and bilirubin were used without further purification.

The pure and mixed films were spread from chloroform solutions (Fisher Chemical Co.) because of the insolubility of bilirubin in the $9: 1 \mathrm{v} / \mathrm{v}$ hexane:ethanol spreading solvents typically used for DPPC and DOPC films $(7,9)$. After solution in chloroform to concentrations of $0.07-0.1 \mathrm{mg} / \mathrm{ml}$, bilirubin was stored in the dark because of its propensity for photo-oxidation. All bilirubinphospholipid-chloroform solutions were made up and used rapidly, always within $8 \mathrm{~h}$. Subphases for film studies were $0.15 \mathrm{M}$ $\mathrm{NaCl}$, or phosphate buffered $\mathrm{NaCl}$ solutions with a total ionic strength of $0.15 \mathrm{M}$. Triply distilled water (9) from all-glass stills was used for all subphases, and for all surface balance cleaning. Experiments were done on the various subphases at $\mathrm{pH} 5.5,7.4$ and 8.0.

All dynamic $\pi$-A experiments were done on a modified Wilhelmy balance described previously $(9,17)$; a submerged "dam" type compression barrier was used for the data of this work. 


\section{RESULTS AND DISCUSSION}

\section{PURE FILM STUDIES}

The behavior of bilirubin in pure films was studied at room temperature $\left(22^{\circ} \mathrm{C}\right)$. The dynamic $\pi$-A results are shown on Figures 1, 2, and 3 for subphase $\mathrm{pH}$ values of 5.5, 7.4, and 8.0, respectively. In these experiments, bilirubin was initially spread from chloroform onto a given subphase to some "apparent" initial concentration, for example $117 \AA^{2} /$ molecule in most experiments (20). The film was then compressed at a rate of $4.6 \mathrm{~min}$ per complete compression-expansion cycle, with an experimental compression ratio of $8: 1$ (maximum area:minimum area).

Figure 1 gives the first and second compression-expansion $\pi$-A isotherms of bilirubin on $0.15 \mathrm{M} \mathrm{NaCl}$ at $\mathrm{pH}=5.5$. It is apparent that at these conditions, bilirubin is a surprisingly surface active molecule, able to reach maximum surface pressures of the order of 63 dynes $/ \mathrm{cm}$. This corresponds to a minimum surface tension of $\sim 10$ dynes $/ \mathrm{cm}$ under the given dynamic compression conditions. This minimum surface tension is only slightly larger than the $\leq 1$ dyne/cm value achieved by pure DPPC films at similar conditions (e.g., 9). It is within the same range of minimum surface tension achieved by lung lavage or extract films of natural pulmonary surfactant (e.g., 7, 13). This high degree of surface activity for bilirubin is at least in part related to its insolubility in water at acidic $\mathrm{pH}$.

Broderson (3) has reported the solubility of bilirubin at various $\mathrm{pH}$ values at $37^{\circ} \mathrm{C}$, and his results display the strong $\mathrm{pH}$ dependence of bilirubin solubility. For example, at $\mathrm{pH} 7$, the solubility of bilirubin at $37^{\circ} \mathrm{C}$ in aqueous Tris buffer (Ionic strength 0.15 M) is given as about $1 \mathrm{nM}$, compared to $\sim 10 \mathrm{nM}$ at $\mathrm{pH} 7.5$ and almost $100 \mathrm{nM}$ at $\mathrm{pH} 8(3)$. These results are consistent with the

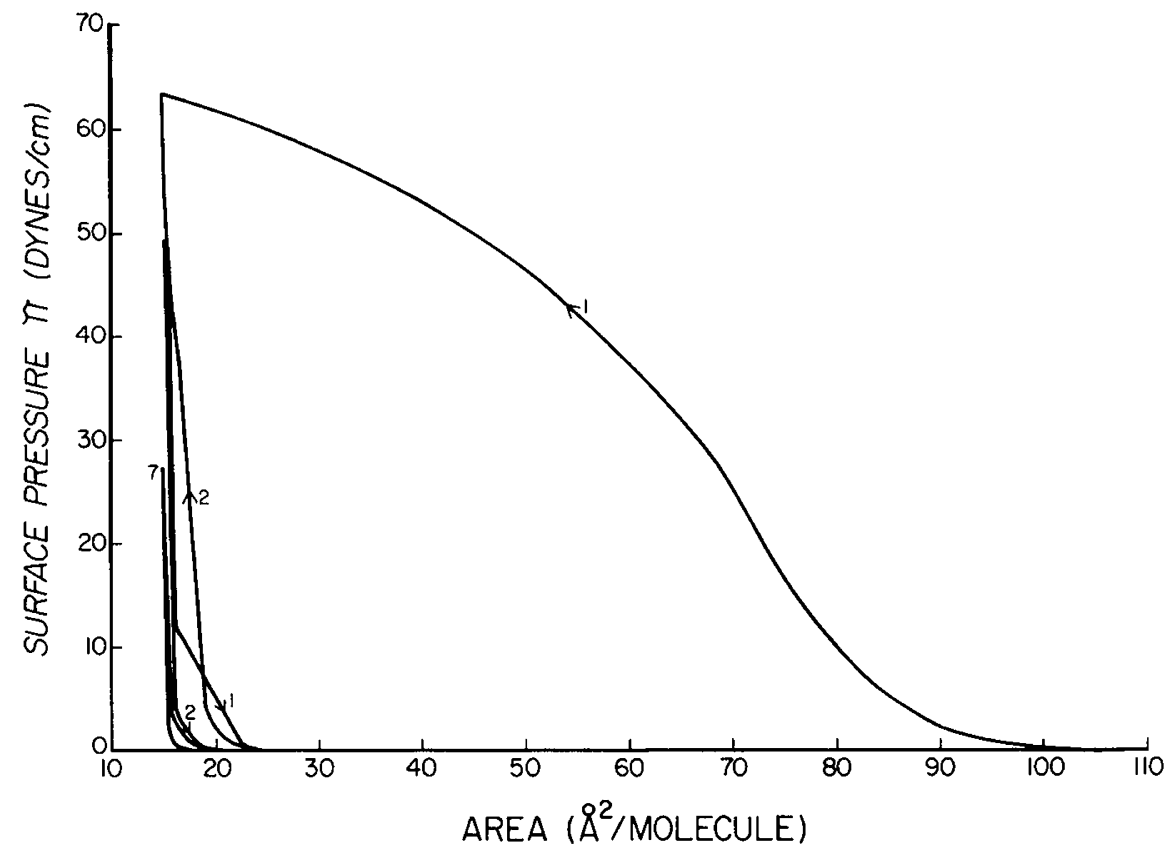

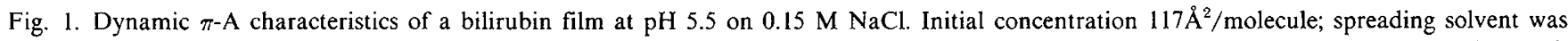
chloroform; cycling speed $4.6 \mathrm{~min} /$ cycle; compression ratio (maximum area:minimum area) $8: 1$; and temperature $=22^{\circ} \mathrm{C}$. First, second and seventh compression-expansion cycles shown as indicated.

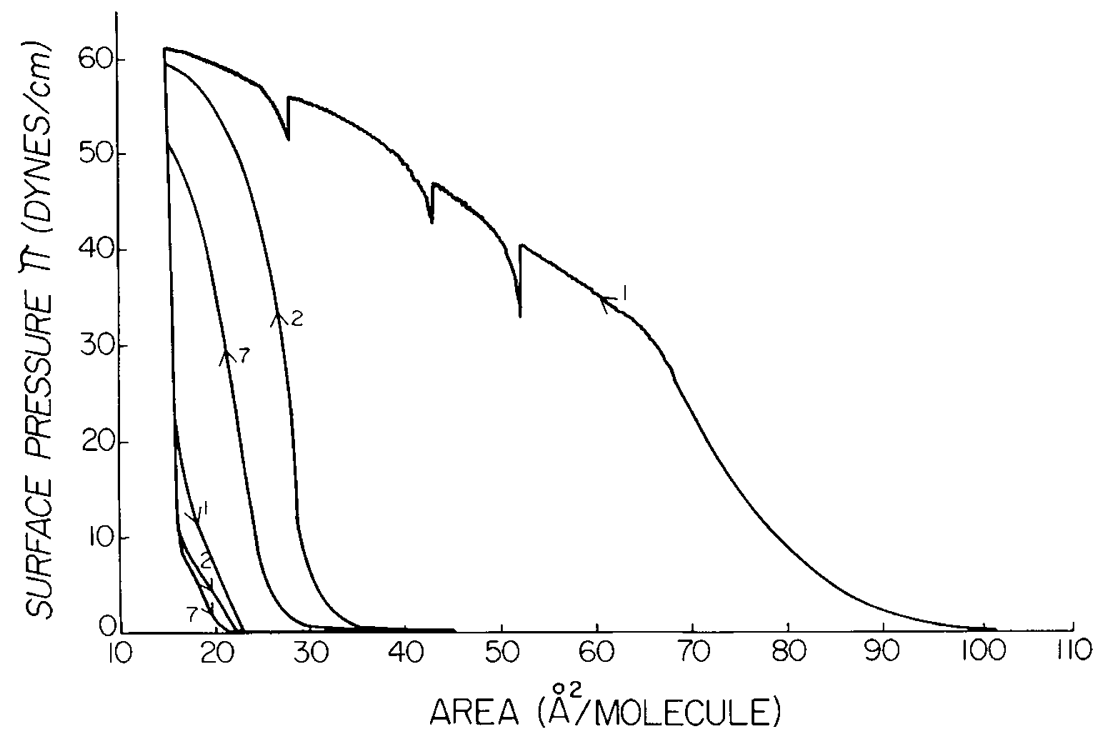

Fig. 2. Dynamic $\pi$-A characteristics of bilirubin at $\mathrm{pH}$ 7.4. Subphase was $0.15 \mathrm{M}$ (ionic strength) phosphate buffered $\mathrm{NaCl}$. Other details as in Figure 1. 


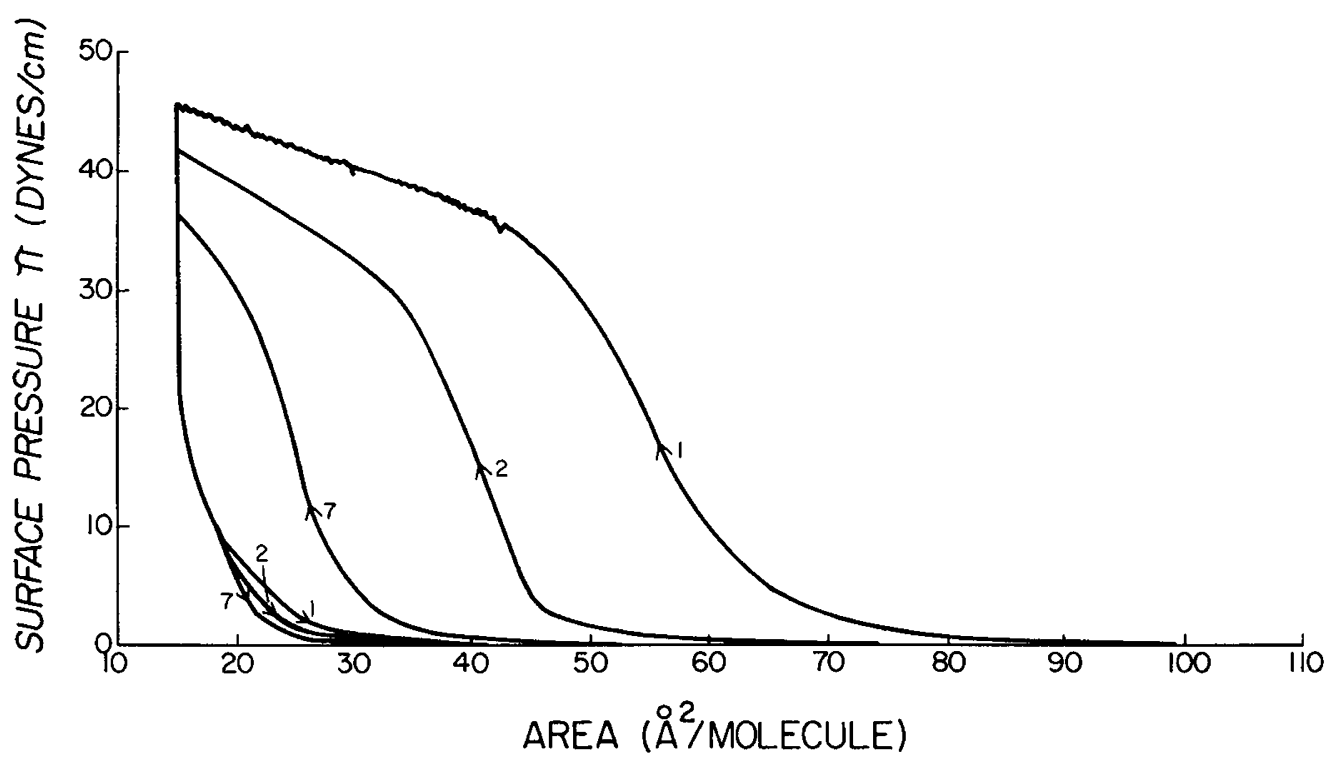

Fig. 3. Dynamic $\pi$-A characteristics of bilirubin at $\mathrm{pH} 8.0$. Other details as in Figure 2.

fact that at acidic $\mathrm{pH}$, the predominant structural form of unconjugated bilirubin is the internally hydrogen-bonded acidic form of the bilirubin IX- $\alpha(Z, Z)$ isomer $(3,4)$. This molecular configuration has decreased attraction for a polar subphase. Consequently, it is able to form surface films capable of exerting high dynamic surface pressures, as seen on the first compression curve of Figure 1.

Another feature of the bilirubin $\pi$-A curves on Figure 1 is that after compression past film collapse, bilirubin is found to be an extremely poor dynamic respeader on subsequent cycles. Consequently, the second compression curve for bilirubin at $\mathrm{pH} 5.5$ is displaced far to the left of the first compression curve, indicating that extensive molecular loss from the surface has occurred in the first compression of the monolayer. This same kind of behavior is found for pure DPPC films compressed past generalized monolayer collapse (9). At pH 5.5, bilirubin is an even worse respreader than DPPC.

Unlike DPPC, bilirubin is a vividly colored molecule in solution. Thus, it can act as a "colorimetric probe" to display the mechanism of the molecular loss that the dynamic $\pi$-A curves of Figure 1 indicate must be occurring between the first and second surface compressions. Specifically, we found that at the $117 \AA^{2} /$ molecule initial concentration condition of Figures 1-3, bilirubin was dilute enough so that only a very faint diffuse tint was initially apparent (to the naked eye) at the liquid-air interface. During the

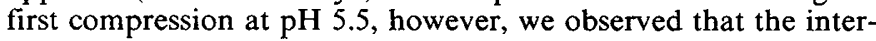
face became strongly tinged orange-brown in color, with thin "ribbons" or straight ridges of greatly increased color aligned relatively parallel to the advancing surface balance barrier. Irregular two dimensional islands of increased color were also seen. A very appealing interpretation of these colored surface structures is that they represent collapse ridges and islands of bilirubin molecules expelled from the monolayer on dynamic compression, and deposited on top of the interface. In particular, the macroscopic ridges bore a striking resemblance to microscopic collapse ridges reported previously in electron micrograph studies by Ries and Kimball (11) and Ries and Walker (12) for the collapse of long chain fatty acid monolayers. Thus, we feel that the lack of dynamic respreading aṕparent for bilirubin at $\mathrm{pH} 5.5$ on Figure 1 is primarily the result of molecules being ejected into surface collapse structures, which maintain their integrity as a collapse phase on top of the interface and fail to respread to a monolayer state on subsequent film expansion.

The dynamic $\pi$-A isotherms for bilirubin films at $\mathrm{pH} 7.4$ and 8.0 on Figures 2 and 3, respectively, seem consistent with the above interpretations. As the $\mathrm{pH}$ is raised, bilirubin aqueous phase solubility increases (3) and the molecule assumes a more open, polar configuration without internal hydrogen bonding. This increased solubility is reflected, for example, in the lower apparent "lift-off" areas of bilirubin films seen at high pH on Figures 2 and 3 compared to the pH 5.5 lift-off of about $105 \AA^{2} /$ molecule shown on Figure 1.

The somewhat "rough" first compression curves of Figures 2 and 3 were observed consistently in our experiments with pure bilirubin films at $\mathrm{pH} 7.4$ and 8.0, particularly the former. The precise location and depth of the sharp breaks shown in the first compression curve of Figure 2 varied from experiment to experiment at $\mathrm{pH}=7.4$, but they were present consistently. A possible explanation for these abrupt breaks in the $\pi$-A isotherm is that they may represent the effects of overstressed regions of bilirubin molecules in the monolayer which suddenly develop enough local energy to be rapidly forced into the subphase during dynamic compression at $\mathrm{pH}$ 7.4. If this is correct, these breaks do not occur at $\mathrm{pH} 5.5$ because bilirubin is so water insoluble that molecules remain in the film until forced into surface collapse fragments or ridges. Similarly, the breaks do not occur as prominently at $\mathrm{pH}$ 8.0 because the subphase solubility of bilirubin is high enough to permit molecules to leave the surface for the subphase in a relatively smooth process during compression. As a result large local stresses do not build up in the monolayer at $\mathrm{pH}$ 8.0.

Changes in maximum surface pressure give another indication that the mode of molecular ejection of bilirubin from a surface film subject to dynamic compression may vary as a function of subphase $\mathrm{pH}$. On Figure 2, bilirubin at $\mathrm{pH} 7.4$ gives rise to a surface pressure of 61 dynes $/ \mathrm{cm}$, whereas the equivalent film at $\mathrm{pH} 8$ only reaches a value of 45 dynes $/ \mathrm{cm}$. This large change in maximum $\pi$ may reflect the fact that at $\mathrm{pH} 8$, the primary collapse mode of bilirubin is into the aqueous subphase rather than into a new collapse phase on top of the interface. This type of behavior would be expected from the increased bilirubin solubility (3).

Aside from maximum surface pressure effects, the data of Figures 1-3 show that the dynamic respreadability of bilirubin on successive surface cycles is increased as the $\mathrm{pH}$ is raised, and the subphase solubility increases. Thus, of the three $\mathrm{pH}$ values inves-

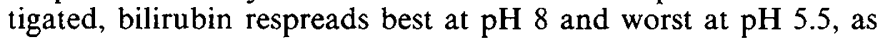
shown by the relative degree of displacement of the second compression isotherms to the left on Figure 1 compared to Figure 3. An interesting speculation from these results is that the mechanism of film collapse, e.g., interfacial region collapse structures on top of the film or sub-surface ejection to the underlying liquid, 
may be one determinant of the ease with which ejected molecules repenetrate to the surface upon film expansion. Mechanistic correlations between specific kinds of film collapse behavior and subsequent dynamic respreadability seem worthy of future study, particularly since this property, among others, is of probable importance for optimal lung surfactant activity $(7,9)$.

\section{INTERACTIONS OF BILIRUBIN WITH DPPC; DOPC}

The behavior of bilirubin in mixed films with DPPC and 9:1 (molar ratio) DPPC:DOPC was examined at $23^{\circ} \mathrm{C}$ as a function of subphase $\mathrm{pH}(21)$. The dynamic $\pi$-A characteristics of DPPC, DOPC, and 9:1 DPPC:DOPC films without bilirubin have been reported by Notter et al. $(8,9)$. In the present study it was verified that these earlier results $(8,9)$ for zwitterionic DPPC and DOPC did not show significant variations between pure water and 0.15 $\mathrm{M} \mathrm{NaCl}$ subphases. Further, it was found that DPPC and 9:1 DPPC:DOPC films do not exhibit strong dependence on $\mathrm{pH}$ in the range $5.5-8.0$ on phosphate buffered saline subphases, in terms of the parameters of film lift-off and collapse plateau ratios on successive surface cycles.

The lack of strong $\mathrm{pH}$ dependence for phosphatidylcholine films does not extend to the case where bilirubin is added as another film component. Figure 4 gives the dynamic $\pi$-A behavior of DPPC:Bilirubin films spread from chloroform to an initial concentration of $\sim 102 \AA^{2} /$ molecule based on total molecules of DPPC plus bilirubin (again refer to ref. 20). Figures $4 a, 4 b$ and $4 c$ are for subphase $\mathrm{pH}$ values of 5.5, 7.4 and 8.0, respectively. Several major features found from Figure 4 are as follows:

(1) Bilirubin shows an interaction with DPPC in the low surface pressure regime, which is reflected in a decreased apparent lift-off in DPPC:Bilirubin films compared to DPPC films. Thus, on Figure 4a, the DPPC:Bilirubin film shows lift-off areas of about $88 \AA^{2} /$ molecule, which is significantly less than the $105 \AA^{2} /$ molecule lift-off found for DPPC films at $\mathrm{pH} 5.5(8,9)$, and also those for pure bilirubin at the same $\mathrm{pH}$ on Figure 1. It appears that at $\mathrm{pH} 5.5$, bilirubin and DPPC exhibit a condensation effect in mixed films at low surface pressure. Note that on Figures $4 b$ and $4 c$, a similar lowered apparent lift-off of $\sim 98 \AA /$ molecule is observed for DPPC:Bilirubin at pH 7.5 and $\mathrm{pH}$ 8. However, the subphase solubility of bilirubin is several orders of magnitude greater at these $\mathrm{pH}$ values than at $\mathrm{pH} 5.5$ (3), and it is more difficult to infer a condensation effect.

The pH 5.5 condensation effect of bilirubin on DPPC films under dynamic compression at low surface pressure has in-
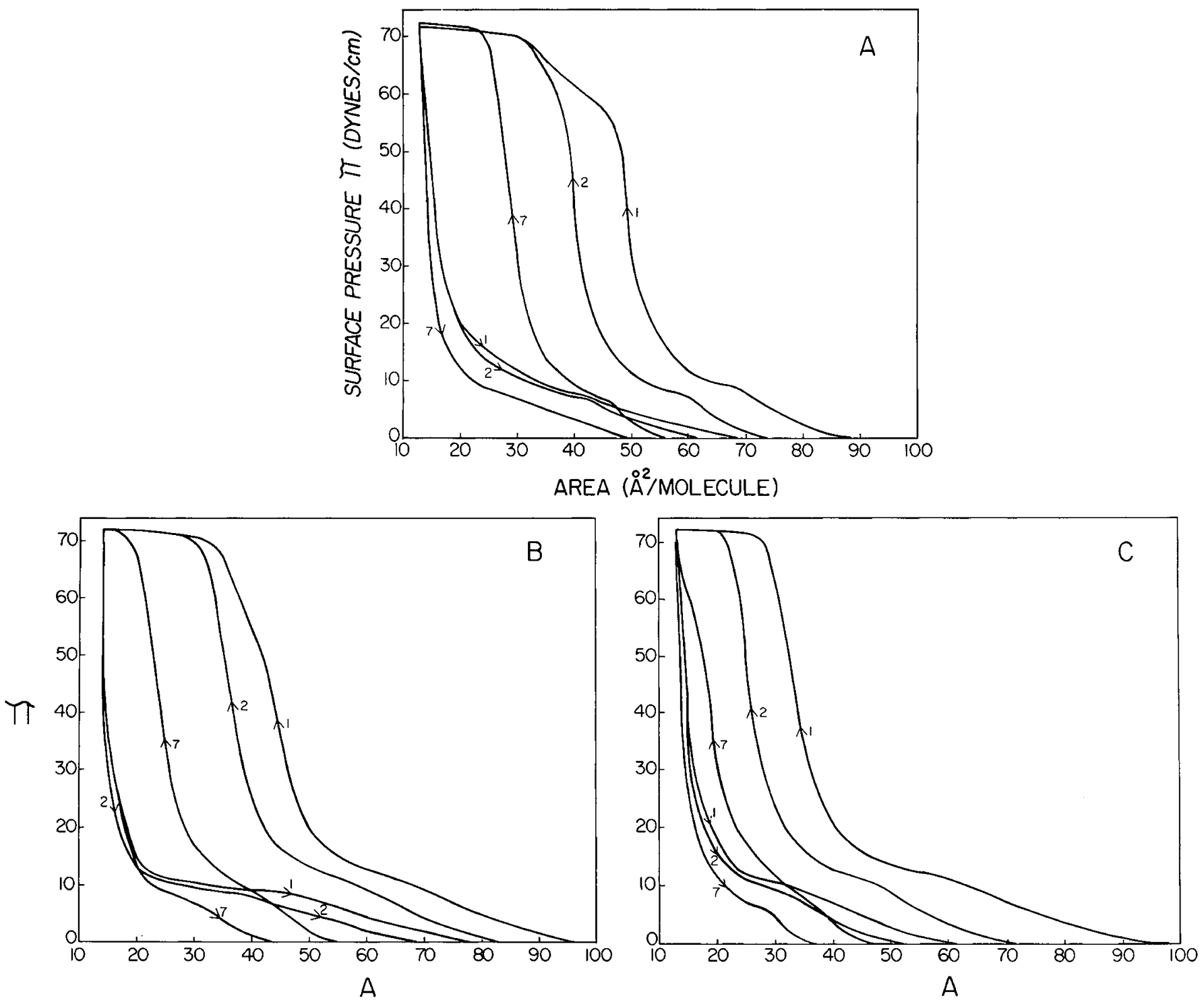

Fig. 4. Effect of bilirubin on DPPC films spread from chloroform. Initial film composition was 7:3 DPPC:Bilirubin. $a$, pH 5.5, $0.15 \mathrm{M}$ NaCl subphase; $b, \mathrm{pH} 7.4$, phosphate buffered $\mathrm{NaCl}$, ionic strength $0.15 \mathrm{M}$; and $c, \mathrm{pH} 8.0$, same subphase as $b$. 
triguing similarities to area effects exerted by cholesterol in binary mixed films with phospholipids. One interpretation of the cholesterol effect, reviewed for example by Cadenhead and Phillips (5) and Shah and Schulman (14), is that the compact structure of cholesterol allows it to fit between phospholipid head groups and tilted fatty acid chains at low surface pressure in such a way as to occupy less area/molecule than predicted by additivity rules from pure film data. Like cholesterol, bilirubin is a ringed molecule. In its acid form, bilirubin is internally hydrogen bonded into a relatively tight structure termed the "knot" structure by Broderson (4). Thus, similar molecular models to those employed by Shah and Schulman (15) for cholesterol with DPPC could explain the apparent bilirubin-DPPC low pressure interactions found present at acid $\mathrm{pH}$ in Figure $4 a$. However, equilibrium measurements, including surface potential-area determinations, are needed to make this definitive.

(2) The DPPC:Bilirubin dynamic $\pi$-A results on Figure 4 also indicate interaction effects in the high surface pressure regime. Further, the magnitude of such effects is greatest if subphase conditions are such that bilirubin is constrained to remain in the surface film at high pressure. Thus, on Figure $4 a$ at $\mathrm{pH} 5.5$, bilirubin acts to increase cycle $2 /$ cycle 1 respreading after compression past collapse in mixed films with DPPC compared to dynamic respreading in pure DPPC films. This is reflected by a smaller shift to the left in the cycle 2 compression curve in Figure $4 a$ compared to what is found for pure DPPC films at similar conditions $(8,9)$. Quantitatively, this increased respreading can be shown from collapse plateau ratio calculations (22); the higher a given collapse plateau ratio is on successive surface cycles, the better the respreading. For the conditions of Figure $4 a$, calculations based on the average of 6 experiments give a cycle 2 /cycle 1 collapse plateau ratio of 0.75 and a cycle $7 /$ cycle $I$ ratio of 0.35 . For comparison, cycle $2 /$ cycle 1 ratios for dilute "lift-off" DPPC films at pH 5.5 are about 0.4 depending on initial concentration variations $(8,9)$, with cycle $7 /$ cycle 1 ratios of slightly less than 0.3 . Thus, the results of Figure $4 a$ show a clear effect of bilirubin in increasing respreadability after collapse in DPPC films at low $\mathrm{pH}$ where the bilirubin is very insoluble in water. This effect of bilirubin on respreading is found to decrease after multiple surface cycles, and effectively disappears by cycle 7 on Figure $4 a$. This decrease after several cycles is consistent with the interpretation that bilirubin is preferentially expelled from the surface compared to DPPC upon each compression past collapse.

The $\pi$-A results of Figure $4 b$ show that at $\mathrm{pH} 7.4$ bilirubin
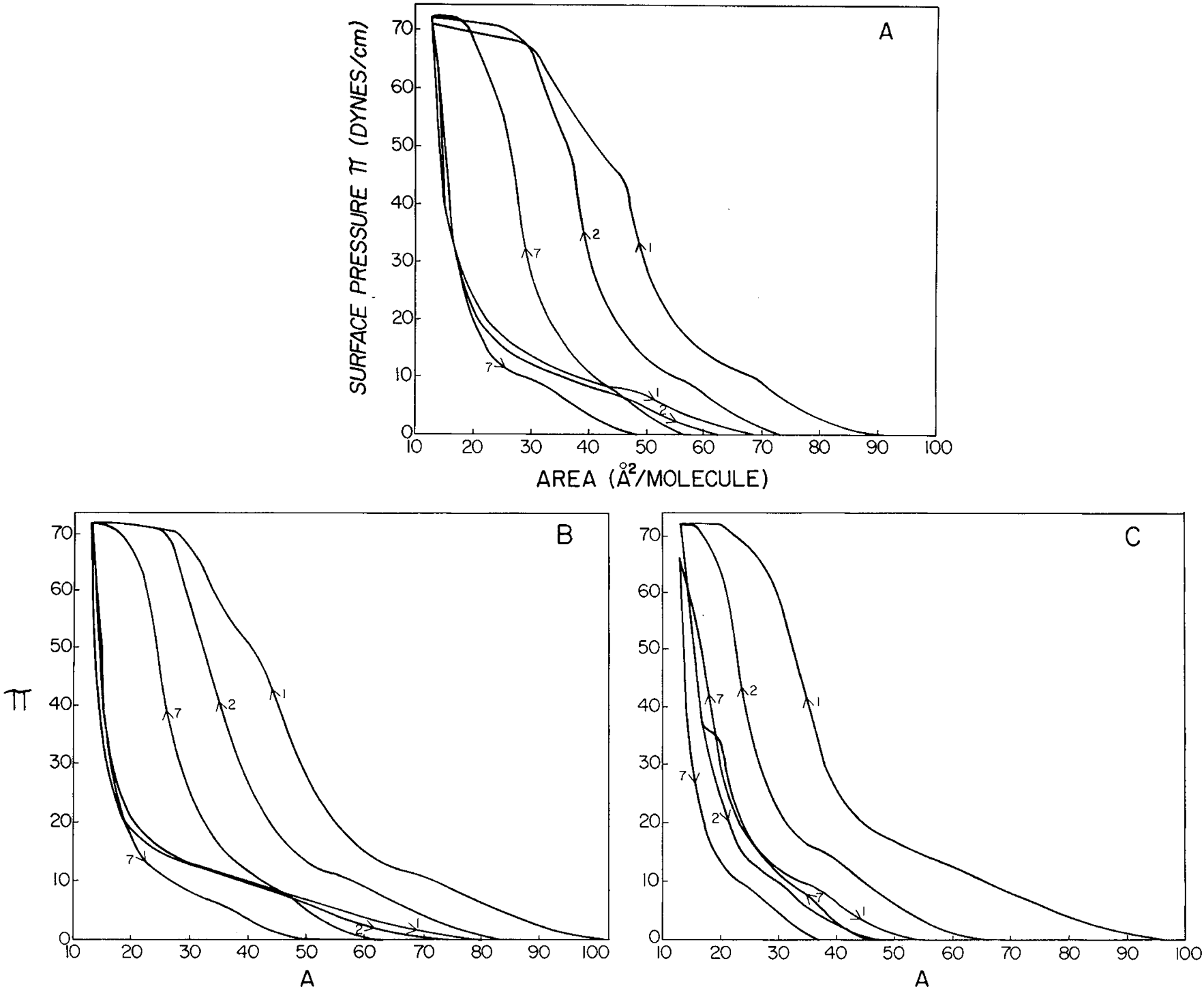

Fig. 5. Effect of bilirubin on 9:1 (Molar Ratio) DPPC:DOPC films. Initial film composition was 61:6:33 DPPC:DOPC:Bilirubin. $a$, pH 5.5, 0.15 M $\mathrm{NaCl}$ subphase; $b, \mathrm{pH} 7.4$, Phosphate Buffered $\mathrm{NaCl}$, ionic strength $0.15 \mathrm{M}$; and $c, \mathrm{pH} 8.0$, same subphase as $b$. 
is able to affect the respreading of DPPC almost as prominently as at pH 5.5. This may be a reflection of the fact that bilirubin is able to generate high surface pressures of $>60$ dynes/cm in pure films at both pH 5.5 and 7.4 (Figs. 1 and 2). It is thus able to remain in the surface at high degrees of film compression. The DPPC:bilirubin results at $\mathrm{pH} 8$ (Fig. $4 c$ ) show that the effect of bilirubin on DPPC respreading after collapse is not impressive on initial surface cycles, presumably because at this $\mathrm{pH}$ bilirubin is unable to remain in the mixed film at high surface pressure.

The results just presented show that, depending upon conditions such as $\mathrm{pH}$, bilirubin exhibits high and low surface pressure interactions with phospholipids in terms of mixed film dynamic cycling behavior. These findings are, in general, consistent with other kinds of data on bilirubin-phospholipid systems. For example, Talafant (18) studied the binding of conjugated and unconjugated bilirubin with phospholipids in various solvents. $\mathrm{He}$ found that lecithins (including DPPC) exhibited the greatest tendency for interaction and aggregate formation. Also, Nagaoka and Cowger (6) investigated bilirubin interaction with lipid liposomes by a fluorescence quenching method and calculated association constants for various systems. They found the highest association constants for bilirubin and sphingomyelin, but also found values nearly as high for bilirubin with phosphatidylcholine and with phosphatidylcholine-5\% cholesterol. These results clearly indicate specific bulk phase interactions of bilirubin and phospholipids. That such interactions also exist in bilirubin-phospholipid films at the liquid-air interface is shown by the results of our monolayer experiments.

In terms of bilirubin interactions with DPPC that would affect surface properties in a way that might be physiologically harmful to lung surfactant activity in vivo, the results of Figure 4 do not suggest a specific concern. In particular, the presence of bilirubin does not change the maximum surface pressure (minimum surface tension) achieved by DPPC surface films. Moreover, dynamic $\pi$ A hysteresis characteristics are not affected in terms of the rapid fall in surface pressure (rise in surface tension) at the start of film expansion. This parameter has recently been put forth as being of physiologic import (10). In fact, the major high surface pressure interaction found for bilirubin with DPPC on Figure 4 is to i.xcrease dynamic respreading at physiologic $\mathrm{pH}$. Such an effect would be beneficial rather than harmful to lung surfactant function.

Lung surfactant is, of course, a complex multicomponent system rather than just a single compound (DPPC). Many researchers feel that of other system components, unsaturated phospholipids (particularly unsaturated phosphatidylcholines and phosphatidylglycerols) may have important functional roles in pulmonary surfactant activity. Consequently, we examined the effect of bilirubin on mixed films composed of $\sim 9: 1 \mathrm{DPPC}$ :DOPC. The results for $\mathrm{pH} 5.5,7.4$ and 8.0 are shown in Figure $5 a, b, c$. The molar ratio of the specific mixed films on Figure 5 when bilirubin is included is 61:6:33 DPPC:DOPC:Bilirubin in terms of the film composition initially spread from chloroform in a given experiment.

The data of Figure 5 again show that bilirubin exerts its greatest effects on phospholipid films in the high surface pressure regime at low $\mathrm{pH}$ where the bilirubin is constrained to stay in the film at or near monolayer collapse. However, these bilirubin interactions are not reflected in any decreased surface tension lowering ability at $\mathrm{pH} 5.5$ or at $\mathrm{pH} 7.4$. In terms of dynamic respreading, collapse plateau ratios calculated from four experiments at the conditions of Figure $5 a(\mathrm{pH} 5.5)$ give average ratios of cycle $2 /$ cycle $1=0.8$ and cycle $7 /$ cycle $1=0.4$. This cycle $2 /$ cycle 1 ratio is slightly higher than the ratio of 0.66 found by Notter et al. (9) for $9: 1$ DPPC:DOPC surface excess films at $23^{\circ} \mathrm{C}$. Also, both $7 / 1$ and $2 /$ 1 ratios are higher than those we found in separate experiments for 9:1 DPPC:DOPC lift-off films ( $\pi$-A curves not shown explicitly). Thus, the data of Figure $5 a$ indicate some dynamic respreading enhancement of bilirubin on DPPC:DOPC films at $\mathrm{pH} 5.5$.

As $\mathrm{pH}$ is increased to physiologic values $(\mathrm{pH}=7.4$, Fig. $5 b$ ), the effect of bilirubin on the $\pi$-A behavior of DPPC:DOPC films decreases from that seen at $\mathrm{pH} 5.5$ in the high surface pressure regime. Average collapse plateau ratios calculated from several $\pi$-A experiments at $\mathrm{pH} 7.4$ were not found to show any large, consistent differences in respreading behavior compared to 9:1 DPPC:DOPC films compressed at similar $\mathrm{pH}$ and initial concentration. The same conclusion generally holds true for the DPPC: DOPC:bilirubin film results of Figure $5 c$.

In summary, this work shows that bilirubin is, by itself, a highly surface active molecule when spread from chloroform at acid $\mathrm{pH}$ where its water solubility is negligible. The degree of surface activity of bilirubin in pure films correlates with subphase solubility, and hence decreases as $\mathrm{pH}$ increases to physiologic values and beyond. At low pH 5.5, bilirubin films collapse at least partially with collapse ridges and lamina on top of the interface. This can be observed by dramatic surface color increases which are apparent to the naked eye. A decreased interfacial color is observed on bilirubin film compression at $\mathrm{pH} 7.4$. No surface color increase is seen at $\mathrm{pH} 8.0$, and the maximum surface pressure generated by bilirubin is markedly decreased. These results seem consistent with the interpretation that at alkaline $\mathrm{pH}$, bilirubin is primarily forced from the interface into the subphase at high degrees of film compression as a result of its increased water solubility.

The interactions of bilirubin in mixed films with important components of lung surfactant (DPPC and DOPC) are found to be most prominent at acidic $\mathrm{pH}$ where the bilirubin is effectively confined to the interface by its low subphase solubility. Bilirubin is found to affect the dynamic $\pi$-A behavior of DPPC films in both the low surface pressure regime (decreased apparent lift-off area), and the high surface pressure regime (increased dynamic respreading after collapse). However, these effects would not seem physiologically detrimental to lung surfactant function per se. The effects decrease as $\mathrm{pH}$ is increased to and beyond physiologic values. Similar but not identical observations apply to results found for bilirubin interactions with 9:1 DPPC:DOPC films.

Our results do not completely rule out a detrimental effect of bilirubin on lung surfactant in vivo. Inasmuch as clear surface interactions are found present in bilirubin-phospholipid films at physiologic $\mathrm{pH}$, the possibility exists that additional effects of bilirubin would be present with more complex lipid films or at different physical conditions. For example, we have not done studies at $37^{\circ} \mathrm{C}$ or studies with bilirubin and extracted or lavaged lung surfactant films. However, on the basis of work to date, there is clearly no rationale for a more conservative approach to the management of hyperbilirubinemia in neonates with RDS.

\section{REFERENCES AND NOTES}

1. Alexander, A. E.: Monolayers of porphyrins and related compounds. J. Chem. Soc., 1813 (1937).

2. Bloomer, J. R., Berk, P. D., Vergalla, J. and Berlin, N. I.: Influence of albumin on the extravascular distribution of unconjugated bilirubin. Clin. Sci. Mol. Med., 45: 517 (1973)

3. Broderson, R.: Bilirubin solubility and interaction with albumin and phospholipid. J. Biol. Chem., 254: 2364 (1979).

4. Broderson, R.: Bilirubin transport in the newborn infant, reviewed with relation to kernicterus. J. Pediatrics, 96: 349 (1980).

5. Cadenhead, D. A. and Phillips, M. C.: Molecular interactions in mixed monolayers. Adv. Chem. Series, 84: 131, American Chem. Soc. (1968).

6. Nagaoka, S. and Cowger, M. L.: Interaction of bilirubin with lipids studied by fluorescence quenching method. J. Biol. Chem., 253: 2005 (1978).

7. Notter, R. H. and Morrow, P. E.: Pulmonary surfactant: a surface chemistry viewpoint. Ann. Biomed. Engr., 3: 119 (1975).

8. Notter, R. H., Holcomb, S. and Mavis, R.: Dynamic surface properties of phosphatidylglycerol-dipalmitoylphosphatidylcholine mixed films. Chem. Phys. Lipids, 27: 305 (1980).

9. Notter, R. H., Tabak, S. A. and Mavis, R. D.: Surface properties of binary mixtures of some pulmonary surfactant components. J. Lipid Res., 21: 10 (1980)

10. Notter, R. H. and Mavis, R. D.: Surface tension hysteresis in lung surfactant films: etiology and physiologic consequences. Pediatr. Res. Abst., 15: 728 (1981)

11. Ries, H. E., Jr., and Kimball, W. A.: Structure of fatty-acid monolayers and a mechanism for collapse. Proc. of 2nd Int'l Congress of Surface Activity. (Academic Press, New York, NY 1957).

12. Ries, H. E., Jr., and Walker, D. C.: Films of mixed horizontally and vertically 
oriented compounds. J. Colloid Interface Sci., 16: 361 (1961)

13. Scarpelli, E. M.: The Surfactant System of the Lung. (Lea and Febiger Philadelphia, PA 1968)

14. Shah, D. O. and Schulman, J. H.: Influence of induced dipoles, metal ions, and cholesterol on the characteristics of phospholipid monolayers. Adv. Chem. Series, 84: 189, American Chem. Soc. (1968).

15. Shah. D. O. and Schulman, J. H.: Influence of calcium, cholesterol, and unsaturation on Lecithin monolayers. J. Lipid Res., 8: 215 (1967).

16. Stenhagen, E. and Rideal, E. K.: The interaction between porphyrins and lipoid and protein monolayers. Biochem. J., 33: 1591 (1939).

17. Tabak, S. A. and Notter, R. H.: Modified technique for dynamic surface pressure and relaxation measurements at the air-water interface. Rev. Sci. Instruments, 48: 1196 (1977).

18. Talafant, E.: Bile pigment-phospholipid interactions. Biochim. Biophys. Acta, 231: 394 (1971).

19. The surface pressure $\pi$ is defined as the surface tension of the pure subphase minus the surface tension when the given surfactant film is present. It is the magnitude of the surface tension lowering generated by the surfactant film.

20. Note that this initial surface concentration, and other film parameters such as the "lift-off" area where surface tension is first affected, are based on a given amount of bilirubin spread as if it all remained at the surface, i.e. the case of negligible subphase solubility is used for calculation simplicity. As discussed in the text, this assumption is not accurate at high $\mathrm{pH}$. In general, the initial concentrations and lift-offs given in the text should be looked upon as reference values for comparative purposes rather than as actual surface concentrations.

21. For all mixed film studies, bilirubin was added to a given phospholipid film at a concentration of close to 36 weight percent of the total surfactant concentration in chloroform solution prior to initial spreading. This high bilirubin concentration was chosen so that maximal interactions would be apparent in mixed films. For the specific DPPC:bilirubin films in Fig. 4, the equivalent molar ratio is 7:3 DPPC:bilirubin.

22. As discussed elsewhere (9), comparison of $\pi$-A curve collapse plateau lengths on successive surface cycles can be used to give a rough measure of molecules ejected from the interface on a given compression that fail to respread on subsequent compressions.

23. Requests for reprints should be addressed to: Dr. R. H. Notter, Division of Neonatology, Department of Pediatrics, University of Rochester School of Medicine, Rochester, New York 14642.

24. This research was supported primarily by NIH grant HL-25170. It was also supported in part by Contract No. DE-AC02-76EV03490 with the U.S. Department of Energy at the University of Rochester Department of Radiation Biology and Biophysics and has been assigned Report No. UR-3490-1938.

25. Received for publication January 7,1981

26. Accepted for publication June 17, 1981. 